\title{
A MEASURE DIFFERENTIAL INEQUALITY WITH APPLICATIONS
}

\author{
R. R. SHARMA
}

ABSTRACT. A measure differential inequality is established and is used to prove a result on the maximum solution, a comparison theorem and a uniqueness theorem of Perron type for abstract measure differential equations.

Introduction. In previous papers [4], [5], the author introduced abstract measure differential equations and studied the problem of the existence of their solutions. As the differential and integral inequalities play a vital role in the theory of differential equations, it is essential to develop certain measure differential inequalities for the further study of (abstract) measure differential equations. This paper is an attempt towards this objective.

In [4], [5], $X$ is taken to be a real or complex linear space with $S_{x}$ and $\bar{S}_{x}$ defined by (1.1) below. It may be noticed, however, that if $X$ is normed and $S_{x}, \bar{S}_{x}$ be defined by (1.2), all the results and proofs in [4], [5] still remain valid. In this paper $S_{x}, \bar{S}_{x}$ would be assumed to be defined by either of the equations (1.1) and (1.2) when $X$ is normed.

1. Notation and preliminaries. Let $X$ be a linear space over the field $\mathcal{F}$ where $\mathcal{F}$ is the set $\mathbf{R}$ of real numbers or the set $\mathbf{C}$ of complex numbers. For each $x \in X$, we define

$$
\begin{array}{lll}
S_{x}=\{a x:-\infty<\alpha<1\}, & \bar{S}_{x}=\{a x:-\infty<a \leq 1\} & \text { if } \mathcal{F}=\mathbf{R} ; \\
S_{x}=\{a x: 0<|\alpha|<1\}, & \bar{S}_{x}=\{a x: 0 \leq|\alpha| \leq 1\} & \text { if } \mathcal{F}=\mathbf{C} .
\end{array}
$$

If $X$ be normed, $S_{x}$ and $\bar{S}_{x}$ may be assumed to have alternative meanings defined by

$$
S_{x}=\{\xi \in X:\|\xi\|<\|x\|\}, \quad \bar{S}_{x}=\{\xi \in X:\|\xi\| \leq\|x\|\} .
$$

Received by the editors September 26, 1973 and, in revised form, December 4, 1973.

AMS (MOS) subject classifications (1970). Primary 34G05; Secondary 46G99.

Key words and phrases. Abstract measure differential equation, measure differential inequality, Radon-Nikodym derivative, total variation measure. 
In the sequel we shall make use of the following elementary properties of the sets defined by (1.1) and (1.2).

Lemma 1. Let $X$ be a real linear space, then

(i) $\bar{S}_{r_{1} x} \subset S_{r_{2} x}$ if and only if one of the inequalities $0<r_{1}<r_{2}$ and $r_{2}<r_{1}<0$ is satisfied;

(ii) $\bar{S}_{x} \subset \bar{S}_{y}$ if and only if there exists a real number $r \geq 1$ such that $y=r x$

(iii) $\left|r_{n}\right| \uparrow|r|$, where $r_{n}$ and $r$ are of the same sign and $r_{n} \neq r$, implies

$$
\bigcup_{n=1}^{\infty} S_{r_{n} x}=\bigcup_{n=1}^{\infty} \bar{S}_{r_{n} x}=S_{r x}
$$

(iv) $\left|r_{n}\right| \downarrow|r|$, where $r_{n}$ and $r$ are of the same sign and $r_{n} \neq r$, implies

$$
\bigcap_{n=1}^{\infty} S_{r_{n} x}=\bigcap_{n=1}^{\infty} \bar{S}_{r_{n} x}=\bar{S}_{r x} .
$$

Proof. (i) if $0<r_{1}<r_{2}$, we have

$$
\bar{S}_{r_{1} x}=\left\{\alpha r_{1} x: \alpha \leq 1\right\}=\left\{\beta x: \beta / r_{1} \leq 1\right\}=\left\{\beta x: \beta \leq r_{1}\right\} \text {; }
$$

and, similarly,

$$
S_{r_{2} x}=\left\{\beta x: \beta<r_{2}\right\}
$$

whence $\bar{S}_{r_{1} x} \subset S_{r_{2} x}$.

If $r_{2} \stackrel{r}{<} r_{1}^{x}<0^{2}$, it is ciear that

$$
\bar{S}_{r_{1} x}=\left\{\beta x: \beta \geq r_{1}\right\}, \quad S_{r_{2} x}=\left\{\beta x: \beta>r_{2}\right\}
$$

and, therefore, $\bar{S}_{r_{1} x} \subset S_{r_{2} x}$.

The converse follows by noting that if $r_{1}$ and $r_{2}$ are not of the same sign, then none of the sets $\bar{S}_{r_{1} x}$ and $S_{r_{2} x}$ is included in the other.

(ii) If $y=r x$ where $r \geq 1$, it follows by (i) that $\bar{S}_{x} \subset \bar{S}_{y}$.

Conversely, assume $\bar{S}_{x} \subset \bar{S}_{y}$. Then for each $\alpha \leq 1$, there exists a $\beta \leq 1$ such that $\alpha x=\beta y$ so that $y=(\alpha / \beta) x$. That $\alpha / \beta \geq 1$ follows from (i).

(iii) Let $0<r_{n}<r$. It follows from (i) that $\bar{S}_{r_{n} x} \subset S_{r x}$. Also $S_{\xi} \subset$ $\bar{S}_{\xi}$ for each $\xi \in X$. Therefore,

$$
\bigcup_{n=1}^{\infty} S_{r_{n} x} \subset \bigcup_{n=1}^{\infty} \bar{S}_{r_{n} x} \subset S_{r x} .
$$


Assume $y \in S_{r x}$. Then $y=\alpha r x$ for some $\alpha<1$. Let $\alpha=1-\epsilon$ where $\epsilon>0$. Since $r / r_{n} \downarrow 1$, there exists an integer $k$ such that $r / r_{k}<1+\epsilon$. Then

$$
\alpha\left(r / r_{k}\right)<(1-\epsilon)(1+\epsilon)=1-\epsilon^{2}<1 ;
$$

and, therefore,

$$
y=\alpha r x=\alpha\left(r / r_{k}\right) r_{k} x \in S_{r_{k} x^{*}}
$$

Thus,

$$
S_{r x} \subset \bigcup_{n=1}^{\infty} S_{r_{n} x} \subset \bigcup_{n=1}^{\infty} \bar{S}_{r_{n} x} .
$$

(1.3) now follows from (1.5) and (1.6).

The proof for the case when $r_{n}<r<0$ is similar.

(iv) Let $\epsilon>0$. Since $r_{n} / r \downarrow 1$, there exists an integer $k$ such that $1<r_{n} / r<1+\epsilon$ for $n \geq k$. Then for each $n \geq k$, we have

$$
\begin{aligned}
r<r_{n}<(1+\epsilon) r & \text { if } r>0 ; \\
(1+\epsilon) r<r_{n}<r & \text { if } r<0 .
\end{aligned}
$$

It follows by (i) that

$$
\bar{S}_{r x} \subset S_{r_{n} x} \subset \bar{S}_{r_{n} x} \subset S_{(1+\epsilon) r x}
$$

for $n \geq k$ and, therefore,

$$
\bar{S}_{r x} \subset \bigcap_{n=1}^{\infty} S_{r_{n} x} \subset \bigcap_{n=1}^{\infty} \bar{S}_{r_{n} x} \subset S_{(1+\epsilon) r x} .
$$

Since $\epsilon$ is arbitrary, it follows that

$$
\bigcap_{n=1}^{\infty} S_{r_{n} x}=\bigcap_{n=1}^{\infty} \bar{S}_{r_{n} x}=\bar{S}_{r x} .
$$

This completes the proof of Lemma 1 .

The following lemmas can similarly be proved.

Lemma 2. If $X$ is a complex linear space, then for each $x \in X$,

(i) $\bar{S}_{\alpha \cdot x}=\bar{S}_{|\alpha| x}$; and $\bar{S}_{\alpha \cdot x} \subset S_{\beta x}$ if and only if $|\alpha|<|\beta|$;

(ii) $\bar{S}_{x} \subset \bar{S}_{y}$ if and only if there exists a complex number $\alpha$ with $|a| \geq 1$ such that $y=a x$

(iii) $\left|\alpha_{n}\right| \uparrow|\alpha|\left(\left|\alpha_{n}\right| \neq|\alpha|\right)$ implies

$$
\bigcup_{n=1}^{\infty} S_{a_{n} x}=\bigcup_{n=1}^{\infty} \bar{S}_{a_{n} x}=S_{a x}
$$


(iv) $\left|\alpha_{n}\right| \downarrow|\alpha|\left(\left|\alpha_{n}\right| \neq|\alpha|\right)$ implies

$$
\bigcap_{n=1}^{\infty} S_{a_{n} x}=\bigcap_{n=1}^{\infty} \bar{S}_{a_{n} x}=\bar{S}_{a x} .
$$

Lemma 3. If $X$ be a normed linear space and $S_{x}, \bar{S}_{x}$ be defined by (1.2), then for each $x \in X$,

(i) $\bar{S}_{\alpha_{x}}=\bar{S}_{|\alpha| x}$; and $\bar{S}_{\alpha_{x}} \subset S_{\beta_{x}}$ if and only if $|\alpha|<|\beta|$;

(ii) $\bar{S}_{x} \subset \bar{S}_{y}$ if and only if there exists a real number $r \geq 1$ such that $\|y\|=r\|x\|$

(iii) $\left\|x_{n}\right\| \uparrow\|x\|\left(\left\|x_{n}\right\| \neq\|x\|\right)$ implies

$$
\bigcup_{n=1}^{\infty} S_{x_{n}}=\bigcup_{n=1}^{\infty} \bar{S}_{x_{n}}=S_{x}
$$

(iv) $\left\|x_{n}\right\| \downarrow\|x\|\left(\left\|x_{n}\right\| \neq\|x\|\right)$ implies

$$
\bigcap_{n=1}^{\infty} S_{x_{n}}=\bigcap_{n=1}^{\infty} \bar{S}_{x_{n}}=\bar{S}_{x} .
$$

Let $S \subset X$, where if $S$ is a proper subset of $X$, it is of the form $S_{\xi}$ for some $\xi \in X$. Let $M$ be a $\sigma$-algebra in $S$ containing all the sets $\bar{S}_{x}^{\xi}$ for all $x \in S$. We shall denote by $c a(S, \mathbb{M})$ the space of all countably additive scalar functions (i.e. real measures or complex measures) on $\mathbb{M}$. Let $\Omega \subset$ $\mathcal{F}$ be defined by

$$
\Omega=\{\alpha:|\alpha|<a\}
$$

Measure differential equations are equations of the form

$$
d \lambda / d \mu=f\left(x, \lambda\left(\bar{S}_{x}\right)\right)
$$

where $\mu$ is a positive $\sigma$-finite measure or a complex measure on $M, d \lambda / d \mu$ is the Radon-Nikodym derivative of a measure $\lambda \in c a(S, M)$ with respect to $\mu$, and $f$ is a function defined on $S \times \Omega$ such that $f\left(x, \lambda\left(\bar{S}_{x}\right)\right)$ is $\mu$-integrable on $S$ for each $\lambda \in c a(S, \mathbb{M})$.

Let $\alpha_{0} \in \Omega, x_{0} \in S, \bar{S}_{x_{0}} \subset X_{0} \in \mathbb{M}$, and let $\mathbb{M}_{0}$ be the smallest $\sigma$-algebra in $X_{0}$ containing $\bar{S}_{x_{0}}-S_{x_{0}}$ and the sets $\bar{S}_{x}$ for each $x \in X_{0}-S_{x_{0}}$. A measure $\lambda \in c a\left(X_{0}, \mathbb{M}_{0}\right)$ is called a solution of (1.8) on $X_{0}$ with initial data $\left[\bar{S}_{x_{0}}, \alpha_{0}\right]$ if $\lambda\left(\bar{S}_{x_{0}}\right)=\alpha_{0}, \lambda(E) \in \Omega$ for $E \in M_{0}, \lambda<<\mu$ on $X_{0}-S_{x_{0}}$

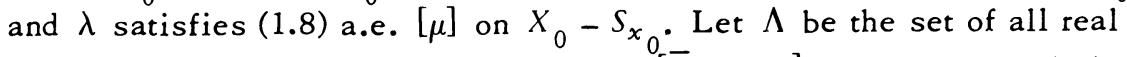
solutions of (1.8) on $X_{0}$ with initial data $\left[\bar{S}_{x_{0}}, a_{0}\right]$. A maximum solution of (1.8) on $X_{0}$ with initial data $\left[\bar{S}_{x_{0}}, \alpha_{0}\right]$ is a solution $\lambda_{0} \in \Lambda$ with the property $\lambda(E) \leq \lambda_{0}(E)$ for each $E \in \mathcal{S}_{0}$ and for each $\lambda \in \Lambda$. 
The total variation measure of a measure $\lambda$ will be denoted by $|\lambda|$.

In this paper we shall be concerned with real solutions only, so we may, and shall, restrict $c a(S, \mathfrak{M})$ to denote the space of real measures. Also, $\mu$ and $f$ will be either real-valued or extended real-valued.

2. A measure differential inequality. The following measure differential inequality is analogous to the differential inequality [2, Theorem 1.2.1, p. 7] and the integral inequality [2, Theorem 5.1.1, p. 315].

Theorem 1. Let $X_{0}=S_{k x_{0}}(k>1)$, and $\lambda, \nu \in c a\left(X_{0}, \mathfrak{M}_{0}\right)$. Suppose that

(i) $\lambda\left(\bar{S}_{x}-S_{x}\right)=\nu\left(\bar{S}_{x}-S_{x}\right)=0$ for each $x \in X_{0}-\bar{S}_{x_{0}}$;

(ii) $\lambda\left(\bar{S}_{x_{0}}\right)<\nu\left(\bar{S}_{x_{0}}\right)$.

Assume further that

(iii) $f(x, a)$ is monotone nondecreasing in a for each fixed $x$;

(iv) $\lambda, \nu \ll \mu$ on $X_{0}-\bar{s}_{x_{0}}$;

(v) the inequalities

$$
\begin{aligned}
& (d \lambda / d \mu)(x) \leq c+f\left(x, \lambda\left(\bar{S}_{x}\right)\right), \\
& (d \lambda / d \mu)(x)>c+f\left(x, \nu\left(\bar{S}_{x}\right)\right),
\end{aligned}
$$

hold a.e. $[\mu]$ on $X_{0}-\bar{S}_{x_{0}}$. Then $\lambda(E)<\nu(E)$ for each $E \in \mathbb{M}_{0}$ such that $|\mu|\left(E-\bar{S}_{x_{0}}\right) \neq 0$.

Proof. Since $X_{0}$ is of the form $S_{k x_{0}}(k>1)$, it follows from the first two properties of Lemmas 1,2, and 3 that each $\bar{S}_{x} \in \mathbb{M}_{0}$ is of the form $\bar{S}_{r x_{0}}$ where $1 \leq r<k$. Suppose that the set $P=\left\{r: \lambda\left(\bar{S}_{r x_{0}}\right) \geq \nu\left(\bar{S}_{r x_{0}}\right)\right.$ is nonempty and $r_{0}=\inf P$. Clearly $r_{0}>1$ by condition (ii), and

$$
\lambda\left(\bar{S}_{r x_{0}}\right)<\nu\left(\bar{S}_{r x_{0}}\right), \quad 1 \leq r<r_{0} .
$$

We shall first show that

$$
\lambda\left(\bar{S}_{r_{0} x_{0}}\right)=\nu\left(\bar{S}_{r_{0} x_{0}}\right) .
$$

Let $r_{n} \uparrow r_{0}\left(1 \leq r_{n}<r_{0}\right)$. It follows by [3, Theorem 1.19(d), p. 16] and property (iii) of Lemmas 1, 2, 3 that

$$
\lim _{n \rightarrow \infty} \lambda\left(\bar{S}_{r_{n} x_{0}}\right)=\lambda\left(S_{r_{0} x_{0}}\right), \quad \lim _{n \rightarrow \infty} \nu\left(\bar{S}_{r_{n} x_{0}}\right)=\nu\left(S_{r_{0} x_{0}}\right) .
$$

(2.1) and condition (i) then imply

$$
\lambda\left(\bar{S}_{r_{0} x_{0}}\right) \leq \nu\left(\bar{S}_{r_{0} x_{0}}\right)
$$


If $r_{0} \in P,(2.2)$ follows by (2.3). If $r_{0} \not \notin P$, there exists a sequence $p_{n} \in P$ such that $p_{n} \downarrow r$. It follows by [3, Theorem 1.19 (e), p. 16] and property (iv) of Lemmas $1,2,3$ that

$$
\lim _{n \rightarrow \infty} \lambda\left(\bar{S}_{p_{n} x_{0}}\right)=\lambda\left(\bar{S}_{r_{0} x_{0}}\right), \quad \lim _{n \rightarrow \infty} \nu\left(\bar{S}_{p_{n^{x}} x}\right)=\nu\left(\bar{S}_{r_{0}{ }_{0}}\right),
$$

whence

$$
\lambda\left(\bar{S}_{r_{0} x_{0}}\right) \geq \nu\left(\bar{S}_{r_{0} x_{0}}\right) .
$$

(2.3) and (2.4) now yield (2.2).

It follows by condition (v) that

$$
\begin{aligned}
& \lambda(E) \leq c \mu(E)+\int_{E} f\left(x, \lambda\left(\bar{S}_{x}\right)\right) d \mu ; \\
& \nu(E)>c \mu(E)+\int_{E} f\left(x, \nu\left(\bar{S}_{x}\right)\right) d \mu
\end{aligned}
$$

for each $E \in \mathbb{M}_{0}$ such that $E \subset X_{0}-\bar{S}_{x_{0}}$ and $(\mu)(E) \neq 0$. Thus, we have

$$
\begin{aligned}
& \lambda\left(\bar{S}_{r_{0} x_{0}}-\bar{S}_{x_{0}}\right) \leq c \mu\left(\bar{S}_{r_{0} x_{0}}-\bar{S}_{x_{0}}\right)+\int_{\bar{S}_{r_{0} x_{0}}-\bar{S}_{x_{0}}} f\left(x, \lambda\left(\bar{S}_{x}\right)\right) d \mu ; \\
& \nu\left(\bar{S}_{r_{0^{x} 0}}-\bar{S}_{x_{0}}\right) \geq c \mu\left(\bar{S}_{r_{0} x_{0}}-\bar{S}_{x_{0}}\right)+\int_{\bar{S}_{r_{0} x_{0}}-\bar{S}_{x_{0}}} f\left(x, \nu\left(\bar{S}_{x}\right)\right) d \mu,
\end{aligned}
$$

where the equality in (2.8) occurs only when $|\mu|\left(\bar{S}_{r_{0} x_{0}}-\bar{S}_{x_{0}}\right)=0$. Since each $\bar{S}_{x}$ for $x \in \bar{S}_{r_{0} x_{0}}-\bar{S}_{x_{0}}$ is of the form $\bar{S}_{r x_{0}}$ where $1<r \leq r_{0}$, it follows by (2.1), (2.2) and condition (iii) that

$$
f\left(x, \lambda\left(\bar{S}_{x}\right)\right) \leq f\left(x, \nu\left(\bar{S}_{x}\right)\right), \quad x \in \bar{S}_{r_{0} x_{0}}-\bar{S}_{x_{0}} .
$$

It now follows from (2.7), (2.8) and (2.9) that

$$
\lambda\left(\bar{S}_{r_{0} x_{0}}-\bar{S}_{x_{0}}\right) \leq \nu\left(\bar{S}_{r_{0} x_{0}}-\bar{S}_{x_{0}}\right) .
$$

Therefore

$$
\lambda\left(\bar{S}_{r_{0} x_{0}}\right) \leq \nu\left(\bar{S}_{r_{0} x_{0}}\right)+\lambda\left(\bar{S}_{x_{0}}\right)-\nu\left(\bar{S}_{x_{0}}\right)<\nu\left(\bar{S}_{r_{0} x_{0}}\right),
$$

by condition (ii). But this contradicts (2.2). Hence the set $P$ is empty and we have

$$
\lambda\left(\bar{S}_{r x_{0}}\right)<\nu\left(\bar{S}_{r x_{0}}\right), \quad 1 \leq r<k
$$

that is,

$$
\lambda\left(\bar{S}_{x}\right)<\nu\left(\bar{S}_{x}\right), \quad x \in X_{0}-S_{x_{0}} .
$$


It now follows from (2.5), (2.6), (2.10) and condition (iii) that $\lambda(E)<\nu(E)$ for $E \in M_{0}$ such that $E \subset X_{0}-\bar{S}_{x_{0}}$ and $|\mu|(E) \neq 0$. This inequality and condition (ii) now yield $\lambda(E)<\nu(E)$ for $E \in \mathbb{M}_{0}$ such that $|\mu|\left(E-\bar{S}_{x_{0}}\right) \neq$ 0 . $\square$

Remark 1. It is obvious from the proof that the inequalities $\leq$ and $>$ in condition ( $v$ ) can also be replaced by $<$ and $\geq$, respectively.

3. Applications. Throughout this section, we shall assume the following conditions to be satisfied:

(i) for each $\xi \in S-\bar{S}_{x_{0}}$, the smallest $\sigma$-algebra containing $\bar{S}_{x_{0}}-$ $S_{x_{0}}$ and $\bar{S}_{x}, x \in \bar{S}_{\xi}-S_{x_{0}}$, is compact in the topology generated by the pseudometric $\rho$ defined by

$$
\rho\left(E_{1}, E_{2}\right)=|\mu|\left(E_{1}-E_{2}\right)+|\mu|\left(E_{2}-E_{1}\right) \quad\left(E_{1}, E_{2} \in \Re\right)
$$

(ii) $|\mu|\left(\bar{S}_{x}-S_{x}\right)=0$ for each $x \in S-S_{x_{0}}$, and $\mu\left(\bar{S}_{x_{0}}\right) \neq 0$;

(iii) $f(x, a)$ is continuous and monotone nondecreasing in $\alpha$ for each fixed $x$.

As an application of Theorem 1, we first prove the following result on the maximum solution.

Theorem 2. Let $\lambda_{0}$ be the maximum solution on $S_{k x_{0}}(k>1)$ of the measure differential equation with the initial condition

$$
d \lambda / d \mu=f\left(x, \lambda\left(\bar{S}_{x}\right)\right), \quad \lambda\left(\bar{S}_{x_{0}}\right)=\alpha_{0} .
$$

Then there exists an $\epsilon_{0}>0$ such that, for $0<\epsilon<\epsilon_{0}$, the solution $\lambda_{\epsilon}$ of

$$
d \lambda / d \mu=f\left(x, \lambda\left(\bar{S}_{x}\right)\right)+\epsilon, \quad \lambda\left(\bar{S}_{x_{0}}\right)=\alpha_{0}+\epsilon
$$

exists on $X_{0}=\bar{S}_{r x_{0}}$ for $r>1$ sufficiently small, and $\lim _{\epsilon \rightarrow 0} \lambda_{\epsilon}(E)=\lambda_{0}(E)$ uniformly on $\mathfrak{M}_{0}$.

Proof. Let $0<\epsilon<a-\left|\alpha_{0}\right|$. It follows from [5, Theorem 1] that there exists a solution $\lambda_{\epsilon}$ of (3.2) on $X_{0}=\bar{S}_{r x_{0}}$ where $r>1$ is such that

$$
\int_{\bar{S}_{r_{0} x_{0}}-S_{x_{0}}}(w(x)+\epsilon) d|\mu|<a-\left|\alpha_{0}\right|-\epsilon .
$$

Since $\lambda_{\epsilon} \ll \mu \ll|\mu|$, and $|\mu|\left(\bar{S}_{x}-S_{x}\right)=0$ for each $x \in S-S_{x_{0}}$, it follows that

$$
\lambda_{\epsilon}\left(\bar{S}_{x}-S_{x}\right)=0, \quad x \in X_{0}-S_{x_{0}}
$$


For $0<\epsilon_{2}<\epsilon_{1} \leq \epsilon$, we have

$$
\begin{gathered}
\lambda_{\epsilon_{2}}\left(\bar{S}_{x_{0}}\right)<\lambda_{\epsilon_{1}}\left(\bar{S}_{x_{0}}\right), \quad\left(d \lambda_{\epsilon_{2}} / d \mu\right)(x)=f\left(x, \lambda_{\epsilon_{2}}\left(\bar{S}_{x}\right)\right)+\epsilon_{2}, \\
\left(d \lambda_{\epsilon_{1}} / d \mu\right)(x)>f\left(x, \lambda_{\epsilon_{1}}\left(\bar{S}_{x}\right)\right)+\epsilon_{2}
\end{gathered}
$$

a.e. $[\mu]$ on $X_{0}-\bar{S}_{x_{0}}$. By Theorem 1, we obtain $\lambda_{\epsilon_{2}}(E)<\lambda_{\epsilon_{1}}(E)$ for each $E \in \mathbb{M}_{0}$ such that $\mu\left(E-\bar{S}_{x_{0}}\right) \neq 0$.

It can be shown, as in the case of $\left\{\lambda_{j}\right\}$ in the proof of [5, Theorem 1], that the family of functions $\left\{\lambda_{\epsilon}\right\}$ is conditionally compact in the space $C\left(\mathbb{M}_{0}\right)$ of all bounded continuous real functions on $\mathfrak{M}_{0}$. Hence there exists a decreasing sequence $\left\{\epsilon_{n}\right\}$ and a function $\lambda_{0} \in C\left(M_{0}\right)$ such that $\epsilon_{n} \rightarrow 0$ and $\lambda_{\epsilon_{n}} \rightarrow \lambda_{0}$ uniformly on $\mathbb{M}_{0}$ as $n \rightarrow \infty$. Clearly, $\lambda_{0}\left(\bar{S}_{x_{0}}\right)=\alpha_{0}$. The continuity of $f$ in $a$ for fixed $x$ implies

$$
\lim _{n \rightarrow \infty} f\left(x, \lambda_{\epsilon_{n}}\left(\bar{S}_{x}\right)\right)=f\left(x, \lambda_{0}\left(\bar{S}_{x}\right)\right)
$$

It then follows by Lebesgue's dominated convergence theorem that

$$
\lim _{n \rightarrow \infty} \int_{E} f\left(x, \lambda_{\epsilon_{n}}\left(\bar{S}_{x}\right)\right) d \mu=\int_{E} f\left(x, \lambda_{0}\left(\bar{S}_{x}\right)\right) d \mu \text {. }
$$

Since $\lambda_{\epsilon_{n}}$ is a solution of (3.2) when $\epsilon=\epsilon_{n}$, we have

$$
\begin{aligned}
\lambda_{\epsilon_{n}}(E) & =\alpha_{0}+\epsilon_{n} \text { for } E=\bar{S}_{x_{0}}, \\
& =\int_{E}\left(f\left(x, \lambda_{\epsilon_{n}}\left(\bar{S}_{x}\right)\right)+\epsilon_{n}\right) d \mu \text { for } E \subset X_{0}-S_{x_{0}}, E \in M_{0} .
\end{aligned}
$$

Thus $\lambda_{0}$ is a solution of (3.1) on $X_{0}$.

We shall now show that $\lambda_{0}$ is the maximum solution of (3.1) on $X_{0}$. Let $\lambda$ be any solution of (3.1). Then

$$
\lambda\left(\bar{S}_{x}-S_{x}\right)=\lambda_{\epsilon}\left(\bar{S}_{x}-S_{x}\right)=0, \quad x \in X_{0}-S_{x_{0}},
$$

since $|\mu|\left(\bar{S}_{x}-S_{x}\right)=0$. Also,

$$
\begin{gathered}
\lambda\left(\bar{S}_{x_{0}}\right)<\lambda_{\epsilon}\left(\bar{S}_{x_{0}}\right), \quad(d \lambda / d \mu)(x)<f\left(x, \lambda\left(\bar{S}_{x}\right)\right)+\epsilon, \\
\left(d \lambda_{\epsilon} / d \mu\right)(x)=f\left(x, \lambda_{\epsilon}\left(\bar{S}_{x}\right)\right)+\epsilon,
\end{gathered}
$$

a.e. $[\mu]$ on $X_{0}-\bar{S}_{x_{0}}$. It follows by Remark 1 that

$$
\lambda(E)<\lambda_{\epsilon}(E)
$$

for all $E \in M_{0}$ such that $|\mu|\left(E-\bar{S}_{x_{0}}\right) \neq 0$. If $|\mu|\left(E-\bar{S}_{x_{0}}\right)=0$, then 


$$
\lambda\left(E-\bar{S}_{x_{0}}\right)=\lambda_{\epsilon}\left(E-\bar{S}_{x_{0}}\right)=0,
$$

since $\lambda, \lambda_{\epsilon} \ll \mu \ll|\mu|$. Thus, if $E \subset X_{0}-\bar{S}_{x_{0}}$, then (3.4) reduces to

$$
\lambda(E)=\lambda_{\epsilon}(E)=0 ;
$$

and if $\bar{S}_{x_{0}} \subset E$, then (3.4) yields

$$
\lambda(E)=\lambda\left(\bar{S}_{x_{0}}\right)<\lambda_{\epsilon}\left(\bar{S}_{x_{0}}\right)=\lambda_{\epsilon}(E) .
$$

By (3.3), (3.5), and (3.6), we obtain $\lambda(E) \leq \lambda_{\epsilon}(E), E \in M_{0}$. The uniqueness of the maximum solution shows that $\lambda_{\epsilon}$ tends uniformly to $\lambda_{0}$ on $\mathbb{M}_{0}$ as $\epsilon \rightarrow 0$. This proves the theorem.

We now prove the following comparison theorem.

Theorem 3. Let $\lambda_{0}$ be the same as in Theorem 2. Let $\nu \in c a\left(X_{0}, M_{0}\right)$ and suppose that $\nu\left(\bar{S}_{x}-S_{x}\right)=0$ for each $x \in X_{0}-\bar{S}_{x_{0}}$, and $\nu\left(\bar{S}_{x_{0}}\right) \leq \lambda_{0}\left(\bar{S}_{x_{0}}\right)$, and the inequality $(d \nu / d \mu)(x) \leq f\left(x, \nu\left(\bar{S}_{x}\right)\right)$ bolds a.e. $[\mu]$ on $X_{0}-\bar{S}_{x_{0}}$. Then $\nu(E) \leq \lambda_{0}(E)$ for each $E \in M_{0}$ such that $|\mu|\left(E-\bar{S}_{x_{0}}\right)>0$.

Proof. Let $\lambda_{\epsilon}$ be any solution of (3.2) on $X_{0}$ for $\epsilon>0$ sufficiently small. Since

$$
\lim _{\epsilon \rightarrow 0} \lambda_{\epsilon}(E)=\lambda_{0}(E) \quad\left(E \in M_{0}\right)
$$

by Theorem 2, it is enough to show that

$$
\nu(E)<\lambda_{\epsilon}(E)
$$

for each $E \in \mathbb{M}_{0}$ such that $|\mu|\left(E-\bar{S}_{x_{0}}\right)>0$.

By assumption, $\nu\left(\bar{S}_{x}-S_{x}\right)=0$. Also, $\lambda_{\epsilon}\left(\bar{S}_{x}-S_{x}\right)=0$, since $\lambda_{\epsilon} \ll|\mu|$. Furthermore,

$$
\begin{gathered}
\nu\left(\bar{S}_{x}\right)<\lambda_{\epsilon}\left(\bar{S}_{x_{0}}\right) ; \quad(d \nu / d \mu)(x)<f\left(x, \nu\left(\bar{S}_{x}\right)\right)+\epsilon, \\
\left(d \lambda_{\epsilon} / d \mu\right)(x)=f\left(x, \lambda_{\epsilon}\left(\bar{S}_{x}\right)\right)+\epsilon
\end{gathered}
$$

a.e. $[\mu]$ on $X_{0}-\bar{S}_{x_{0}}$. The inequality (3.7) now follows by Theorem 1. $\square$ The above theorem can be applied to prove the following uniqueness theorem of Perron type.

Theorem 4. Suppose that $g(x, \alpha)$ is defined on $X_{0} \times \Omega, g\left(x, \nu\left(\bar{S}_{x}\right)\right)$ is $\mu$-integrable on $X_{0}$ for each $\nu \in c a\left(X_{0}, M_{0}\right), g(x, 0)=0, g(x, \alpha)$ is monotone nondecreasing in a for each fixed $x$, and $\nu=0$ is the only solution of the equation 


$$
d \nu / d|\mu|=g\left(x, \nu\left(\bar{S}_{x}\right)\right), \quad \nu\left(\bar{S}_{x_{0}}\right)=0 .
$$

Assume further that

$$
\left|f\left(x, \alpha_{1}\right)-f\left(x, \alpha_{2}\right)\right| \leq g\left(x,\left|\alpha_{1}-\alpha_{2}\right|\right)
$$

for all $\left(x, a_{1}\right),\left(x, a_{2}\right) \in X_{0} \times \Omega$. Then there exists at most one solution of (3.1) on $X_{0}$.

Proof. Let $\lambda_{1}, \lambda_{2}$ be two solutions of (3.1) on $X_{0}$. Define

$$
\lambda(E)=\left|\lambda_{1}-\lambda_{2}\right|(E) \quad\left(E \in \mathbb{m}_{0}\right) .
$$

Then $\lambda$ is a positive measure on $\Re_{0}$, and

$$
\begin{aligned}
\lambda(E) & =0 \text { for } E=\bar{S}_{x_{0}}, \\
& =\int_{E}\left|f\left(x, \lambda_{1}\left(\bar{S}_{x}\right)\right)-f\left(x, \lambda_{2}\left(\bar{S}_{x}\right)\right)\right| d|\mu| \text { for } E \subset X_{0}-S_{x_{0}}, E \in \mathbb{M}_{0} .
\end{aligned}
$$

By (3.9), we obtain

$$
\begin{aligned}
\lambda(E) & \leq \int_{E} g\left(x,\left|\lambda_{1}\left(\bar{S}_{x}\right)-\lambda_{2}\left(\bar{S}_{x}\right)\right|\right) d|\mu| \\
& =\int_{E} g\left(x,\left|\left(\lambda_{1}-\lambda_{2}\right)\left(\bar{S}_{x}\right)\right|\right) d|\mu| \\
& \leq \int_{E} g\left(x,\left|\lambda_{1}-\lambda_{2}\right|\left(\bar{S}_{x}\right)\right) d|\mu|=\int_{E} g\left(x, \lambda\left(\bar{S}_{x}\right)\right) d|\mu| .
\end{aligned}
$$

Then it follows by Theorem 3 that $\lambda(E) \leq \nu_{0}(E)$ for each $E \in \mathbb{M}_{0}$ such that $|\mu|\left(E-\bar{S}_{x_{0}}\right)>0$, where $\nu_{0}$ is the maximum solution of (3.8). But $\nu=0$ is the only solution of (3.8). Therefore $\lambda(E)=0$ for each $E \in \mathbb{M}_{0}$ such that $|\mu|\left(E-\bar{S}_{x_{0}}\right)>0$. If $|\mu|\left(E-\bar{S}_{x_{0}}\right)=0$, it is clear by (3.10) that $\lambda\left(E-\bar{S}_{x_{0}}\right)$ $=0$. Thus, if $E \subset X_{0}-\bar{S}_{x_{0}}$, then $\lambda(E)=0$, and if $\bar{S}_{x_{0}} \subset E$, then again

$$
\lambda(E)=\lambda\left(\bar{S}_{x_{0}}\right)+\lambda\left(E-\bar{S}_{x_{0}}\right)=0 .
$$

Hence $\lambda(E)=0$ for each $E \in \mathbb{M}_{0}$. This proves $\lambda_{1}=\lambda_{2}$ and, hence, the theorem.

\section{REFERENCES}

1. N. Dunford and J. T. Schwartz, Linear operators. I: General theory, Pure and Appl. Math., vol. 7, Interscience, New York, 1958. MR 22 \#302.

2. V. Lakshmikantham and S. Leela, Differential and integral inequalities. Vol. I, Math. in Sci. and Engineering, vol. 55-I, Academic Press, New York, 1969.

3. W. Rudin, Real and complex analysis, McGraw-Hill, New York, 1966. MR $35 \# 1420$. 
4. R. R. Sharma, An abstract measure differential equation, Proc. Amer. Math. Soc. 32 (1972), 503-510. MR 45 \#691.

5. - Existence of solutions of abstract measure differential equations, Proc. Amer. Math. Soc. 35 (1972), 129-136. MR 46 \#3943.

DEPARTMENT OF MATHEMATICS, REGIONAL INSTITUTE OF TECHNOLOGY, JAMSHEDPUR, INDIA 